\title{
VECTOR EXTRAPOLATED FAST ML ALGORITHMS FOR EMISSION TOMOGRAPHY
}

\author{
N. RAJEEVAN
}

\author{
Department of Electrical Engineering \\ Indian Institute of Science \\ Bangalore 560012 , INDIA
}

\section{ABSTRACT}

A new class of fast cyclic iterative algorithms for maximum likelihood estimation of emission densities in ECT is developed. The Approach is based on acceleration of convergence by vector extrapolation. The minimal polynomial and the reduced rank extrapolation techniques are integrated with the iterations in EM and EM search algorithms. The resulting algorithms, tested with simulated phantoms, have shown improved convergence and have given very encouraging results.

\section{METHOD}

In this paper we develop a new class of fast cyclic iterative algorithms for emission density estimation based on convergence acceleration by vector extrapolation. In this approach a finite number of consecutive estimates produced by gradient based algorithms, such as EM or EM search, is considered as a sequence of vectors generated by an operator. These vectors -re then extrapolated in the vector space to obtain a new estimate of the emission density. This density vector is then used as the next starting estimate for the gradient based algorithm from which a finite set of iterates are again generated and vector extrapolated to obtain the next emission density estimate. This process of cyclic iteration is continued till acceptable convergence is achieved.

Two methods of vector extrapolation, the Minimal Polynomial Extrapolation (MPE) and the Reduced Rank Extrapolation (RRE), are implemented and tested using simulated phantoms. In MPE, the extrapolated estimate is obtained as a weighted sum of the sequence of estimates generated by the gradient based algorithm, where the weights are related to the coefficients of the minimal polynomial of the operator. In RRE a weighted sum of the correction vectors is used to obtain the extrapolated estimate. These algorithms are briefly outlined here and detailed in [ i].

These two approaches, the MPEML and the RREML algorithms, are implemented and tested using simulated phantoms. Fig. 1 shows the loglikelihood functions estimated using the proposed algorithms and compares them with those estimated using the EM and EMS algorithms. Fig. 2 gives the reconstructed images using [VIPEML and RREML algorithms with both EM and EMS as base algorithms.

$$
\text { REFERENCE }
$$

[1] Rajeevan N., et.al., "Vector extrapolated fast ML algorithms for Emission Tomography," submitted to IEEE Trans. Medical Imaging.
MPEML Algorithm Initialisation

$\lambda^{\delta}=$ strictly positive initial estimate $\mathrm{m}=$ degree of minimal polynomial (taken as a small positive integer) MPEML $n=0$, cycle index

do

$\left\{\right.$ generate $\lambda^{k}, k=1, \ldots, m$ using gradient based algorithm compute $\Delta\left(\lambda^{k}\right)=\lambda^{k+1} \lambda^{k}, k=0, \ldots, m-1$ find the coefficient vector, $c$, of the minimal polynomial from

$$
\underline{c}=-[\Delta]^{+} \Delta\left(\lambda^{m}\right), c_{m}=1.0
$$

$$
\begin{aligned}
& \lambda^{n, m}=\sum w_{k} \cdot \lambda^{k} \\
= & \lambda^{n, m} \quad ; n+t \quad ;
\end{aligned}
$$

3 until convergence.

$\lambda$ is the MPEML estimate of the emission density.

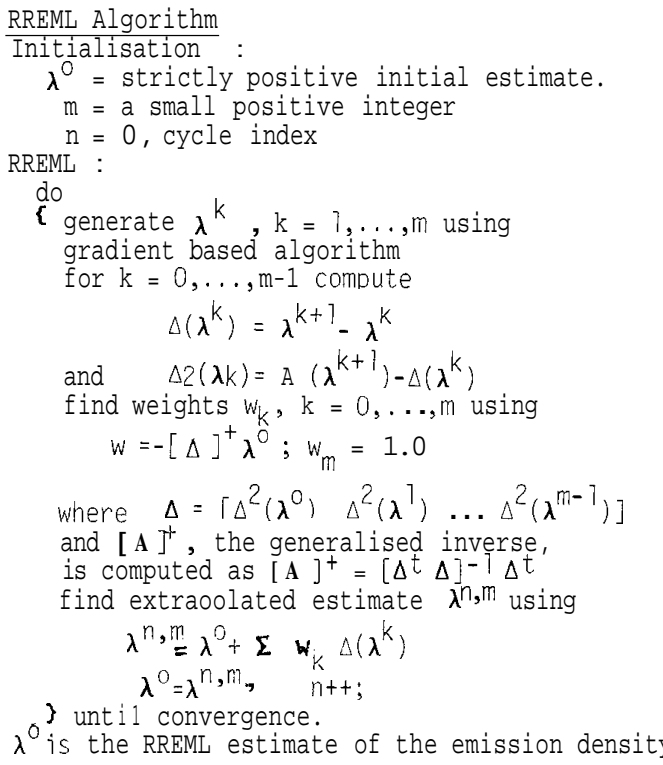

0370 Annual International Conference of the IEEE Engineering in Medicine and Biology Society, Vol. 12, No. 1, 1990 

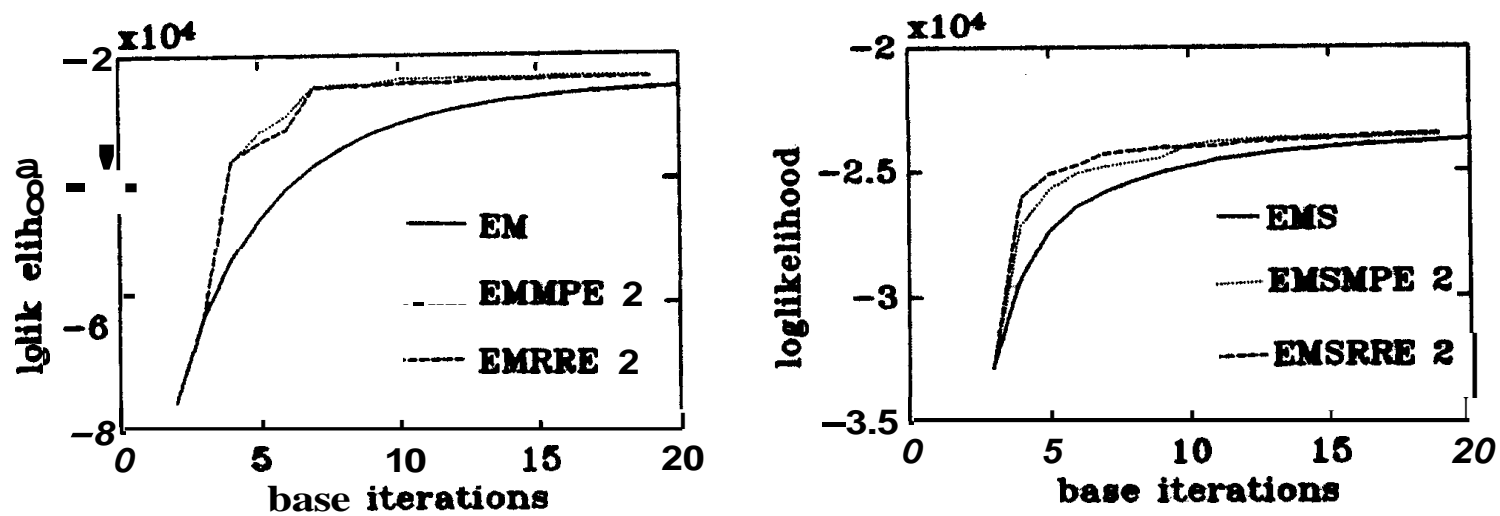

Fig. 1 Plot of loglikelihood vs base iterations.

a) EM - EM algorithm

EMMPE 2 - MPE algorithm with two EM iterations per cycle

EMRRE 2 - RRE algorithm with two EM iterations per cycle

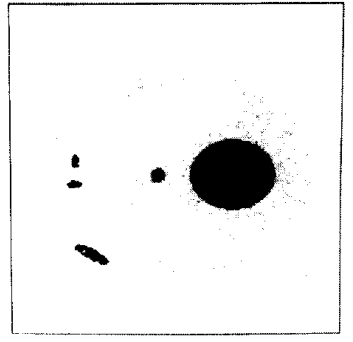

phan box

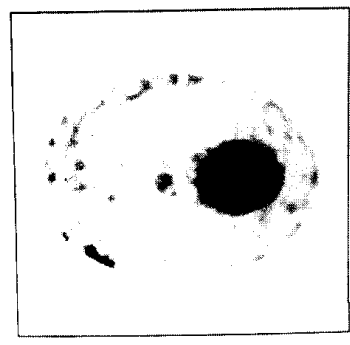

$\operatorname{ems} 10$

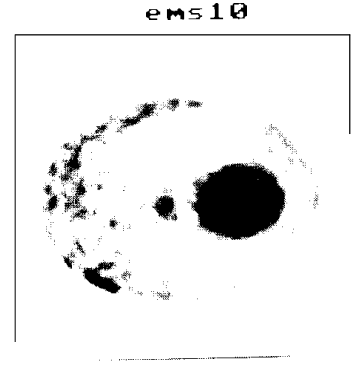

ems rom $2-2$

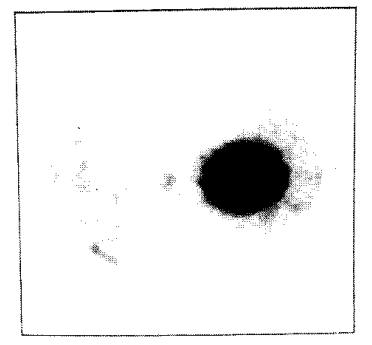

emis

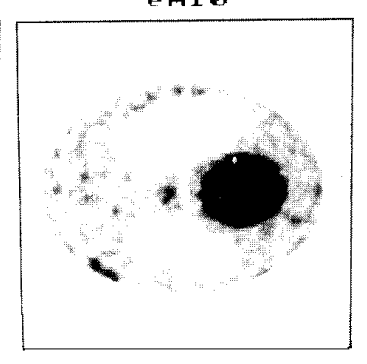

$\operatorname{en} 20$

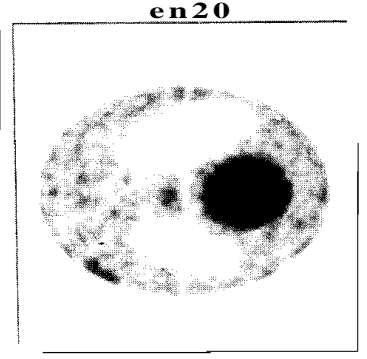

emrre $2-3$

b) EMS - EM search algorithm

EMSMPE 2 - MPE algorithm with two EMS iterations per cycle

EMSRRE 2 - RRE algorithm with two EMS iterations per cycle
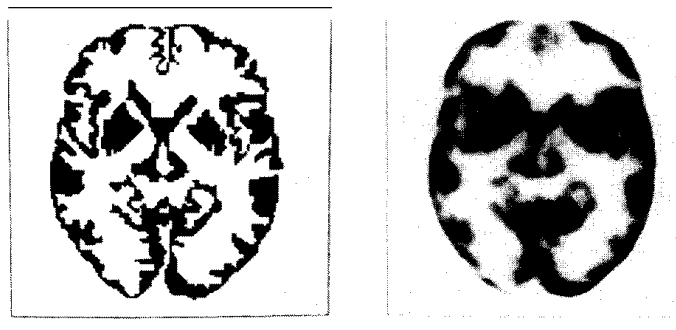

brain original

EMIO
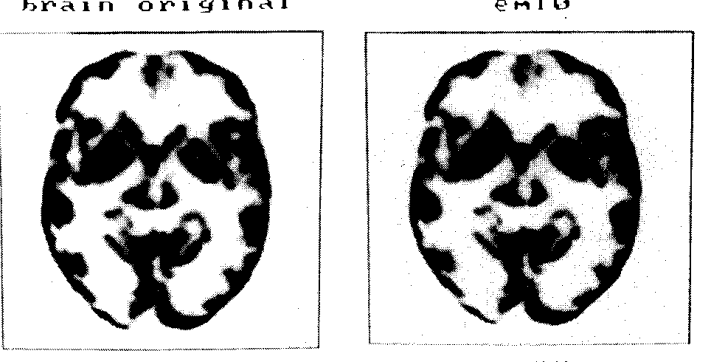

ems 10

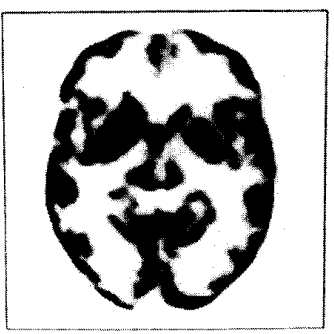

emsmpe 22

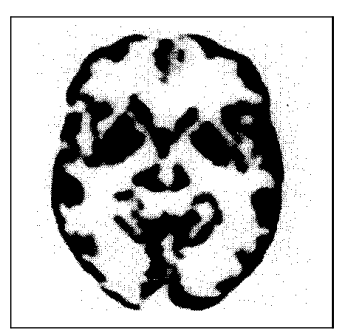

eminpe 2-3

Fig. 2 Reconstructed images using MPEML and RREML algorithms.

EM10 : 10 iterations of EM algorithm; EMS10 : 10 iterations of EM Search algorithm; EMPRE 2-3:

3 cycles of EMPRE with 2 iterations per cycle; EMSRRE 2-2:2 cycles of EMSRRE with 2 iterations per cycle; EMMPE 2-3:3 cycles of EMMPE with 2 iterations per cycle; EMSMPE 2-2:2 cycles of EMSMPE with 2 iteratioons per cycle.

Annual International Conference of the IEEE Engineering in Medicine and Biology Society, Vol. 12, No. 1, 1990 